11,12

\title{
Влияние границ зерен на распределение компонентов в бинарных сплавах
}

\author{
(C) П.Е. ЛЬвов ${ }^{1}$, В.В. Светухин ${ }^{1,2}$ \\ ${ }^{1}$ Ульяновский государственный университет, \\ Ульяновск, Россия \\ ${ }^{2}$ Институт нанотехнологий микроэлектроники РАН, \\ Москва, Россия \\ E-mail: LvovPE@sv.uven.ru
}

(Поступила в Редакцию 4 апреля 2017 г. В окончательной редакции 16 мая 2017 г.)

На основе метода функционала плотности свободной энергии (уравнения Кана-Хилларда) разработана феноменологическая модель, описывающая влияние границ зерен на распределение компонентов в бинарных сплавах. Модель основывается на предположении о различии параметров взаимодействия между компонентами твердого раствора в объеме и на границе зерна. На основе спектрального метода предложена разностная схема для решения уравнения Кана-Хилларда с параметрами взаимодействия, зависящими от координат. Для различных соотношений параметров взаимодействия в объеме и на границе зерна, температуры, а также состава сплава модель может приводить к различным типам распределения растворенного компонента: обеднение или обогащение зернограничной области, преимущественная зернограничная преципитация, конкурентная преципитация в объеме и на границе зерна и др.

Работа выполнена в рамках выполнения государственного задания Минобрнауки России и проекта РФФИправительство Ульяновской области № 16-42-732113.

DOI: 10.21883/FTT.2017.12.45244.113

\section{1. Введение}

Твердые растворы на основе металлов (сплавы) имеют поликристаллическую структуру, т.е. состоят из небольших фрагментов (зерен), имеющих различную ориентацию друг относительно друга. Области соприкосновения между соседними зернами формируют границы зерен, которые представляют собой дефект кристаллической структуры, оказывающий существенное влияние на различные процессы, протекающие в сплавах [1,2]. Границы зерен оказывают заметное влияние на процессы пластической деформации [2,3], рекристаллизации [2], перераспределения примесей и дефектов [2,4] и др. Таким образом, как макро-, так и микроскопические свойства сплавов в значительной мере определяются процессами, протекающими вблизи границ зерен.

Процесс формирования границ зерен крайне сложен, что обусловлено нарушением упорядоченности в расположении атомов [2]. В связи с этим значительное количество работ посвящено созданию моделей самих границ $[1,4,5]$, а также целому ряду других задач: описанию миграции границ зерен, в том числе в условиях механических нагружений [6,7], эволюции дислокационной структуры [8], зернограничной сегрегации [9-11], влияния размера зерна на распределение фаз [12] и др.

Одной из важных задач прикладного материаловедения является изучение влияния границ зерен на распределение компонентов твердого раствора. В литературе известен ряд попыток описания данного процесса с помощью уравнения диффузии [13-16] и на основе метода Монте-Карло [17,18]. В настоящей работе предполагается рассмотреть влияние границ зерен на распределение компонентов сплава (включая формирование фаз) в бинарных сплавах на основе метода функционала плотности свободной энергии [19-21]. Данный подход наиболее часто используется для описания фазовых переходов в системах, не имеющих структурных дефектов, как в области метастабильных [22-24], так и нестабильных [25,26] состояний. Этот метод обычно применяется в предположении безграничности рассматриваемой среды, при этом влияние эффектов, связанных с наличием границ зерен, как правило, не рассматривается. Вместе с тем хорошо известно, что границы зерен могут приводить к локальному изменению скорости диффузии атомов растворенного компонента (см. например, $[1,2])$, а также энергии взаимодействия между компонентами твердого раствора [5,11,27], что неизбежно сказывается на характере их распределения внутри зерна. Коэффициенты диффузии и параметры взаимодействия между компонентами твердого раствора вблизи границ зерен крайне сложно определить экспериментальными методами. Поэтому важной является разработка моделей фазовых переходов с учетом влияния границ зерен, с целью установить связь между распределением фаз и характеристиками сплава на атомном масштабе вблизи границ зерен (например, энергиями взаимодействия, коэффициентами диффузии и др.). 


\section{2. Основные приближения модели}

Рассмотрим бинарный сплав, состоящий из атомов сортов $A$ и $B$. Для определенности будем полагать, что атомы сорта $A$ принадлежат растворителю, атомы сорта $B-$ растворенному веществу. Пусть данный сплав удовлетворяет приближению регулярного раствоpa, тогда для однородного, безграничного кристалла энергия смешения в расчете на одну частицу имеет вид $g \equiv g\left(c_{B}\right)$ :

$$
g\left(c_{B}\right)=\Omega c_{A} c_{B}+k_{B} T\left[c_{B} \ln c_{B}+c_{A} \ln c_{A}\right],
$$

где $T-$ температура, $k_{B}-$ постоянная Больцмана, $c_{A}$ и $c_{B}$ - концентрации атомов растворителя и растворенного вещества соответственно $\left(c_{A}+c_{B}=1\right)$, $\Omega=\frac{z}{2}\left(2 g_{A B}-g_{A A}-g_{B B}\right)-$ параметр квазихимического взаимодействия, $g_{A B}, g_{A A}, g_{B B}-$ энергии парного взаимодействия между атомами соответствующих сортов, $z$ - число ближайших соседей.

Расстояние между атомами, находящимися вблизи границ, несколько отличается от расстояния в объеме зерна (см. например, $[5,11]$ ). Это неизбежно приводит к отличию энергий парного взаимодействия $\left(g_{A B}, g_{A A}, g_{B B}\right)$ между атомами в объеме и вблизи границы зерна. Кроме того, изменение межатомного расстояния приводит к изменению поля упругих напряжений, плотность энергии которого в простейшем приближении [21] также описывается квадратической функцией состава. Таким образом, будем считать, что параметр квазихимического взаимодействия $\Omega$ является некоторой эффективной величиной, зависящей от координат, которая в первом приближении [21] учитывает также и упругие напряжения.

В более общем случае, когда вблизи границ существенно изменяется плотность раствора, решение задачи о влиянии границ зерен на распределение компонентов сплава необходимо проводить с привлечением нескольких параметров порядка.

Зависимость параметра квазихимического взаимодействия $\Omega$ от координат, к сожалению, неизвестна, поэтому мы рассмотрим особенности распределения компонентов сплава между зернограничной областью и объемом зерна на основе нескольких естественных предположений для функции $\Omega(x, y, z)$. Во-первых, вдали от границ зерен значение $\Omega$ должно быть равным $\Omega_{0}$, определяемому для макроскопических фрагментов твердого раствора. Во-вторых, значение параметра $\Omega$ определяется расстояниями до одной или нескольких границ зерна, т. е. точки, равноудаленные от границ зерен, имеют одинаковые значения параметра $\Omega$. Таким образом, поверхности постоянного значения $\Omega(x, y, z)=$ const должны соответствовать геометрии самого зерна.

Отличие параметра квазихимического взаимодействия в объеме зерна $\Omega_{0}$ от значения на границе $\Omega_{G B}$ будем характеризовать величиной $\Delta=\left(\Omega_{G B}-\Omega_{0}\right) / \Omega_{0}$.

Рассмотрим фрагмент бинарного твердого раствора кубической формы $\left(L_{x} \times L_{y} \times L_{z}\right)$, который будет представлять собой модель зерна. Для описания зависимости параметра $\Omega$ от координат может быть использована степенная функция, удовлетворяющая сделанным выше предположениям

$$
\begin{gathered}
\Omega(x, y, z)=\Omega_{0} \\
\times\left[1+\frac{\Delta}{R_{\Omega}}\left\{\left|2 x-L_{x}\right|^{m}+\left|2 y-L_{y}\right|^{m}+\left(2 z-\left.L_{z}\right|^{m}\right\}\right]\right. \\
R_{\Omega}=L_{x}^{n}+L_{y}^{n}+L_{z}^{n},
\end{gathered}
$$

где $n$ - действительное число, которое связано с шириной переходного слоя $\delta$ и размером зерна $L$. С помощью линейной аппроксимации параметра $\Omega$ в одномерном случае можно получить оценку для ширины переходного слоя $\delta \sim L(2 n)^{-1}$. Данную формулу также можно было бы использовать и для оценки параметра $n$, если определить с помощью иных методов (например, метода молекулярной статики) характерное расстояние $\delta$, на котором происходит изменение параметров взаимодействия между компонентами твердого раствора.

Наряду со степенной зависимостью (2) удобными для моделирования могли бы быть также кусочнонепрерывная функция координат или функция, описывающая экспоненциальное убывание параметра взаимодействия по мере удаления от границы зерна.

Обычно в термодинамике твердого состояния рассматриваются сплавы с параметрами взаимодействия, не зависящими от координат, что позволяет сравнительно просто анализировать фазовое равновесие для бинарных сплавов. В общем случае фазы определяются как части системы, которые существуют одновременно в равновесии друг с другом, соприкасаясь между собой [28]. При заданных температуре и давлении необходимым условием равновесия является постоянство химического потенциала [28], что обеспечивает отсутствие потоков компонентов раствора (см. например, [21,29]). Заметим, что использование данного определения для систем с параметрами взаимодействия, зависящими от координат, допускает наличие неоднородности образующихся фаз по составу.

В рассматриваемом случае параметры взаимодействия зависят от координат, поэтому установить фактические равновесные составы фаз и границы метастабильности можно только по результатам численного расчета распада рассматриваемого твердого раствора. Вместе с тем некоторые качественные рассуждения о влиянии границ зерен на процесс распределения растворенного компонента могут быть проведены на основе анализа фазовых диаграмм, соответствующих параметрам взаимодействия в объеме $\Omega_{0}$ и на границе зерна $\Omega_{G B}=\Omega_{0}(1+\Delta)$. На рис. 1 приведены фазовые диаграммы, рассчитанные с помощью плотности свободной энергии смешения (1) для значений параметра квазихимического взаимодействия $\Omega_{0}$ и $\Omega_{G B}$ (при $\Delta=0.5$ и $\Delta=-0.2$ ). В качестве безразмерной температуры $T^{*}$ выбрано отношение $T^{*}=2 k_{B} T / \Omega_{0}$, при этом критическая температура для объема зерна соответствует $T_{C}^{*}=1$, а для границы зерна $T_{C G B}^{*}=1+\Delta$. 

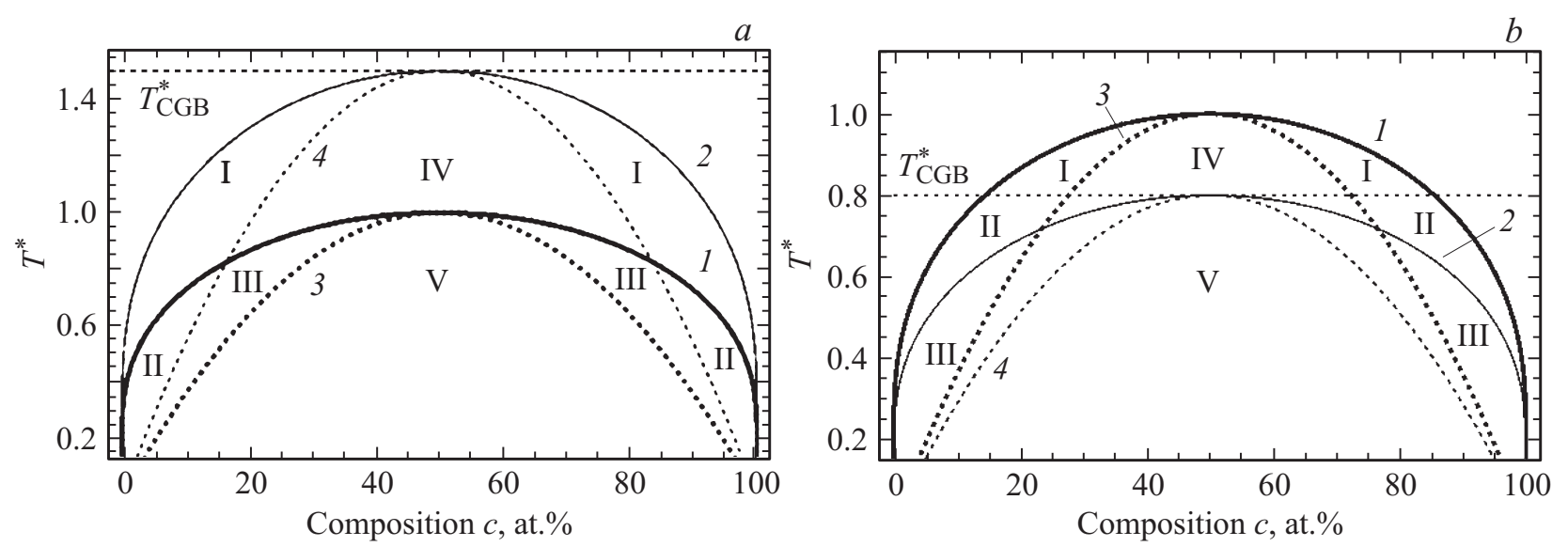

Рис. 1. Фазовые диаграммы, описываемые параметрами взаимодействия в объеме зерна и в зернограничной области. Сплошными линиями на частях $a(\Delta=0.5)$ и $b(\Delta=-0.2)$ отображены линии бинодали: 1 - для объема зерна, $2-$ для границы зерна. Пунктирными линиями отображен предел метастабильности: 3 - для объема зерна, 4 - для границы зерна. Горизонтальной пунктирной линией обозначена критическая температура для границы зерна $T_{C G B}^{*}=1+\Delta$.

В случае, если $\Delta>0$, энергия смешения на границе зерна становится выше, чем в его объеме. Очевидно, что это должно приводить к возникновению потока атомов растворенного компонента от границы зерна в его объем. При этом вблизи границы зерна должна образовываться область, обедненная по атомам сорта $B$. C другой стороны, степень пересыщения вблизи границы зерна оказывается заметно выше (рис. 1,a), чем в объеме, что приводит к уменьшению работы зародышеобразования [30,31]. Кроме того, для границы зерна предел метастабильности смещается в область меньших составов, что приводит к возрастанию в данной области флуктуаций, дисперсия которых может быть определена в виде $[19,28]$

$$
\sigma^{2}=k_{B} T v_{0}\left(\left.V \frac{\partial^{2} g}{\partial c^{2}}\right|_{c_{M}}\right)^{-1}
$$

где $V$ - объем сплава, для которого проводится вычисление состава, $v_{0}$ - объем, приходящийся на один атом, $c_{M}-$ состав сплава. Таким образом, в случае $\Delta>0$, несмотря на отток атомов сорта $B$, можно ожидать наличия режима, связанного с преимущественным образованием выделений второй фазы вблизи границ зерен. Следует заметить, что в отдельных случаях зернограничная область может оказаться в области нестабильных состояний, для которых реализуется безактивационное образование второй фазы так, как это происходит в процессе спинодального распада.

В случае, если $\Delta<0$, энергия смешения на границе окажется меньше, чем в объеме зерна, тогда следует ожидать возникновения потока вещества из матрицы на границу зерна, т. е. должно происходить обогащение зернограничной области атомами растворенного компонента. Вместе с тем изменение параметра взаимодействия будет приводить (см. рис. $1, b$ ) к снижению критической температуры $T_{C G B}^{*}$ и степени пересыщения, а также к увеличению состава, соответствующего границе метастабильности. Таким образом, формирование выделений второй фазы на границе зерна может быть затруднено несмотря на увеличение концентрации атомов сорта $B$.

В отношении рассматриваемой модели необходимо сделать ряд важных замечаний. Во-первых, в рассматриваемой системе происходит обмен веществом между объемом и зернограничной областью. Поэтому перераспределение вещества может приводить к сдвигу по составу состояний вблизи границы зерна (рис. $1, a$ и $b$ ) как в сторону больших, так и меньших концентраций. Кроме того, вблизи границы зерна происходит монотонное изменение параметра $\Omega$ от $\Omega_{0}$ до $\Omega_{G B}$, что означает наличие монотонного изменения растворимости атомов сорта $B$ в матрице и предела метастабильности по мере приближения к границе зерна. В связи с этим проведенное деление на области различных участков фазовых диаграмм (рис. 1, $a$ и $b$ ) является условным. Во-вторых, в рассматриваемой модели зерна в форме куба имеются локальные области, где влияние оказывают несколько границ. Эти области (вблизи ребер и вершин куба) характеризуются наибольшим отклонением параметра взаимодействия от значения в объеме. В некоторых режимaх, например, зернограничной сегрегации данные области могут быть наиболее эффективными стоками для атомов растворенного компонента и играть доминирующую роль в перераспределении вещества внутри зерна. B-третьих, можно ожидать также и существования различных смешанных режимов распада, для которых механизмы формирования второй фазы в объеме и на границе зерна могут существенно отличаться друг от друга.

Таким образом, с точки зрения рассмотренной модели возможными вариантами влияния границ зерен на перераспределение состава сплава являются: обогащение или обеднение зернограничной области, преимущественная зернограничная или объемная преципитация растворенного компонента, а также конкурентная преципитация, 
допускающая образование выделений второй фазы как в объеме зерна, так и на его границе. Изменение параметров взаимодействия вблизи границ зерен, по всей видимости, может влиять и на процесс спинодального распада, который наблюдается в области нестабильных состояний.

\section{3. Уравнение Кана-Хилларда с параметрами взаимодействия, зависящими от координат}

Рассмотрим распад бинарного сплава с учетом влияния границ зерен на основе метода функционала плотности свободной энергии. Пусть свободная энергия сплава описывается в виде функционала [19,31]:

$$
G=n_{0} \int_{v}\left(g\left(c_{B}\right)+\frac{1}{2} \kappa\left(\nabla c_{B}\right)^{2}\right) d V,
$$

где $g\left(c_{B}\right)$ - свободная энергия смешения в расчете на одну частицу, определяемая формулой (1), $\kappa$ коэффициент градиентной энергии, $c_{B} \equiv c(\mathbf{r}, t)-$ концентрация растворенного компонента, $n_{0}$ - количество атомов в единице объема. В соответствии с предположениями, сделанными в разделе 2 , будем считать, что параметр квазихимического взаимодействия $\Omega$ зависит от координат. Каном и Хиллардом [31] (см. также [32]) было показано, что коэффициент $\kappa$ линейно зависит от параметра взаимодействия $\Omega$, что может быть отражено в виде соотношения

$$
\kappa=\kappa_{0} \Omega a^{2},
$$

где $\kappa_{0}-$ постоянная величина, определяемая потенциалом взаимодействия между компонентами твердого раствора, $a$ - период решетки. Таким образом, в рамках рассматриваемой модели параметр $\kappa$ также оказывается функцией координат.

Получим уравнение Кана-Хилларда с учетом зависимости параметров $\Omega$ и $\kappa$ от координат.

Для концентрационного поля $c_{B}(\mathbf{r}, t)$ выполняется уравнение непрерывности

$$
\frac{\partial c_{B}}{\partial t}=- \text { divj. }
$$

где поток ј определяется в виде

$$
\mathbf{j}=-M \nabla \mu,
$$

$M-$ подвижность, $\mu-$ химический потенциал, который определяется как функциональная производная в виде

$$
\mu=\frac{\delta G}{\delta c_{B}}=\frac{\partial I}{\partial c_{B}}-\sum_{i=1}^{3} \frac{\partial}{\partial x_{i}}\left(\frac{\partial I}{\partial\left(\partial c_{B} / \partial x_{i}\right)}\right) .
$$

Здесь $I$ - подынтегральная функция в формуле (4), $x_{i}$ - координаты радиус-вектора $\mathbf{r}$. Теперь уравнение (5), описывающее эволюцию концентрационного поля с учетом переменных параметров $\Omega$ и $\kappa$, принимает вид:

$$
\frac{\partial c_{B}}{\partial t}=n_{0} \nabla\left[M \cdot \nabla\left(\frac{\partial g}{\partial c_{B}}-\nabla\left(\kappa \nabla C_{B}\right)\right)\right] .
$$

Уравнение (6) при неизменных $\Omega$ и $\kappa$ трансформируется в уравнение Кана-Хилларда для среды с постоянными параметрами взаимодействия [21].

Для дальнейшего рассмотрения уравнения (6) удобно перейти от переменной $c_{B}$ к параметру порядка $\eta=c_{B}-c_{A}=2 c_{B}-1$. Теперь, в предположении постоянства подвижности $M$, уравнение (6) принимает вид

$$
\frac{\partial \eta}{\partial \tau}=\nabla^{* 2}\left[\phi-\nabla^{*}\left(\kappa^{*} \nabla^{*} \eta\right)\right]
$$

где

$$
\kappa^{*}=\frac{\kappa}{\Omega_{0} a^{2}}, \tau=t \frac{n_{0} M \Omega_{0}}{a^{2}}, \nabla^{*}=a \nabla,
$$

$a$ - период решетки, $\phi-$ безразмерная функция, которая определяется формулой

$$
\phi \equiv-2 \Omega^{*} \eta+T^{*} \ln \left[\frac{1+\eta}{1-\eta}\right]
$$

где использовано обозначение $T^{*}=2 k_{B} T / \Omega_{0}$, $\Omega^{*}=\Omega(x, y, z) / \Omega_{0}$.

\section{4. Численные методы решения уравнения Кана-Хилларда с параметрами взаимодействия, зависящими от координат}

Одним из наиболее эффективных методов решения уравнения Кана-Хилларда является спектральный метод, основанный на использовании быстрого преобразования Фурье. Данный метод решения уравнения КанаХилларда достаточно хорошо развит для случая постоянной подвижности [33] и подвижности, зависящей от локального состава сплава [34] (см. также, [25,26]). Чтобы применить данный метод к разработанной модели, его необходимо адаптировать для случая, когда параметр квазихимического взаимодействия $\Omega$, а вместе с ним и параметр $\kappa$, зависят от координат.

В случае переменного параметра взаимодействия разностная схема для Фурье-образа параметра порядка $\hat{\eta}_{k}^{n}$ в момент времени $\tau_{n}=n \Delta \tau$ принимает вид:

$$
\hat{\eta}_{\mathbf{k}}^{n+1}=\hat{\eta}_{\mathbf{k}}^{n}-\Delta \tau|\mathbf{k}|^{2}\left\{\hat{\phi}_{\mathbf{k}}^{n}-i \mathbf{k} \mathscr{F}_{\mathbf{k}}\left[\kappa_{0} \Omega^{*} \mathscr{F}_{\mathbf{k}^{\prime}}^{-1}\left[i \mathbf{k}^{\prime} \hat{\eta}_{\mathbf{k}^{\prime}}^{n}\right]\right]\right\},
$$

где $\mathscr{F}_{\mathbf{k}}[\cdot]$ и $\mathscr{F}_{\mathbf{k}^{\prime}}^{-1}[\cdot]$ - прямое и обратное преобразования Фурье соответственно, $\hat{\phi}_{\mathbf{k}}^{n}-$ Фурье-образ производной от плотности свободной энергии в момент времени $\tau_{n}, \mathbf{k}$ и $\mathbf{k}^{\prime}$ - волновые векторы.

Для повышения устойчивости разностной схемы (8) необходимо провести ее расщепление с помощью элементов $\kappa_{0}\left(\Omega^{*}-\widetilde{\Omega}\right) \mathscr{F}_{\mathbf{k}^{\prime}}^{-1}\left[i \mathbf{k}^{\prime} \hat{\eta}_{\mathbf{k}^{\prime}}^{n}\right]$ и $\kappa_{0} \widetilde{\Omega}_{\mathbf{k}^{\prime}}^{-1}\left[i \mathbf{k}^{\prime} \hat{\eta}_{\mathbf{k}^{\prime}}^{n+1}\right]$, что 
позволяет получить окончательные формулы для моделирования распада твердого раствора, параметры взаимодействия которого зависят от координат

$$
\begin{aligned}
\hat{\eta}_{\mathbf{k}}^{n+1}=\hat{\eta}_{\mathbf{k}}^{n} & -\frac{\Delta \tau|\mathbf{k}|^{2}}{1+\Delta \tau \kappa_{0} \tilde{\Omega}|\mathbf{k}|^{4}} \\
& \times\left\{\hat{\phi}_{\mathbf{k}}^{n}-i \mathbf{k} \mathscr{F}_{\mathbf{k}}\left[\kappa_{0} \Omega^{*} \mathscr{F}_{\mathbf{k}^{\prime}}^{-1}\left[i \mathbf{k}^{\prime} \hat{\eta}_{\mathbf{k}^{\prime}}^{n}\right]\right]\right\}
\end{aligned}
$$

Константа $\widetilde{\Omega}$ выбирается из соображений устойчивости разностной схемы (9). В данной работе параметр $\widetilde{\Omega}$ принимался равным единице.

\section{5. Параметры моделирования и начальные условия}

Процесс перераспределения компонентов твердого раствора будем рассматривать для зерна в виде куба с ребром $L=128 a\left(L_{x}=L_{y}=L_{z}=L\right)$. Параметр $\kappa_{0}$ примем равным 0.2. При использовании разностной схемы (9) шаг по времени $\Delta \tau$ будем считать равным $5 \cdot 10^{-4}$.

Характерное расстояние, на котором происходит изменение параметров взаимодействия, составляет порядка нескольких периодов решетки [11]. Для аппроксимации зависимости $\Omega(x, y, z)$ используем зависимость степенного типа (2). Предположим, что характерное расстояние, на котором происходит изменение параметра взаимодействия, составляет $\delta \sim 4 a$, тогда показатель степени в формуле (2) можно оценить как $n \sim L /(2 \delta)=16$.

Начальное состояние сплава будем описывать в виде случайного гауссова поля с математическим ожиданием $\langle c\rangle=c_{M}$. При определении дисперсии для области метастабильных состояний будем считать ее локальной характеристикой, которая изменяется при приближении к границе зерна в соответствии с формулой (3). При рассмотрении образования фаз в области нестабильных состояний, когда формула (3) неприменима, будем считать среднеквадратичное отклонение $\sigma$ равным 0.01 . Если в сплаве, в силу зависимости параметров взаимодействия от координат, одновременно присутствуют области, относящиеся к метастабильным и нестабильным состояниям (например, область III на рис. 1, $a$ ), то дисперсия для расчета начального распределения считалась всюду постоянной величиной, совпадающей со значением в объеме зерна.

\section{6. Результаты моделирования}

Для выявления различных типов распределения вещества между объемом зерна и зернограничной областью рассмотрим эволюцию концентрационного поля для описанной выше системы при нескольких составах $c_{M}$, температурах $T^{*}$ и параметрах $\Delta$, которые определяют характер распада твердого раствора и относятся к различным участкам фазовых диаграмм, приведенных на рис. $1, a, b$.

6.1. Обогащение и обеднение зерногранич н о й о блас ти. На рис. 2 приведены результаты расчета распределения состава в бинарном сплаве $\left(T^{*}=0.85\right.$, $c_{M}=16$ at.\%, $\left.\Delta=0.5\right)$, соответствующем области I на рис. 1, $a$. В этом случае зародышеобразование как в объеме, так и на границе зерна отсутствовало. Различие во взаимодействии приводит к перераспределению компонентов твердого раствора, в результате которого зернограничная область обедняется по атомам сорта $B$ (см. рис. 2, кривые $1-3$ ). При этом эффект обеднения

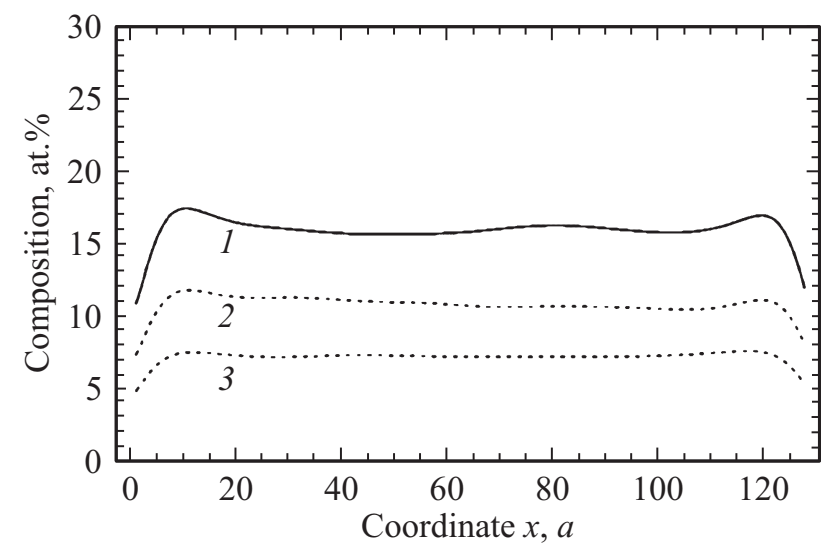

Рис. 2. Концентрационные профили в бинарном сплаве $\left(c_{M}=16\right.$ at.\%, $\left.T^{*}=0.85\right)$ в момент времени $\tau=30$ в случае обеднения зернограничной области. Сплошная линия 1 соответствуют концентрационному профилю вдоль оси $x$ для $y=z=L / 2$. Пунктирные линии взяты вблизи границы зерна: 2 - посередине грани куба $(y=L, z=L / 2), 3$ - вдоль ребра куба $(y=z=L)$.

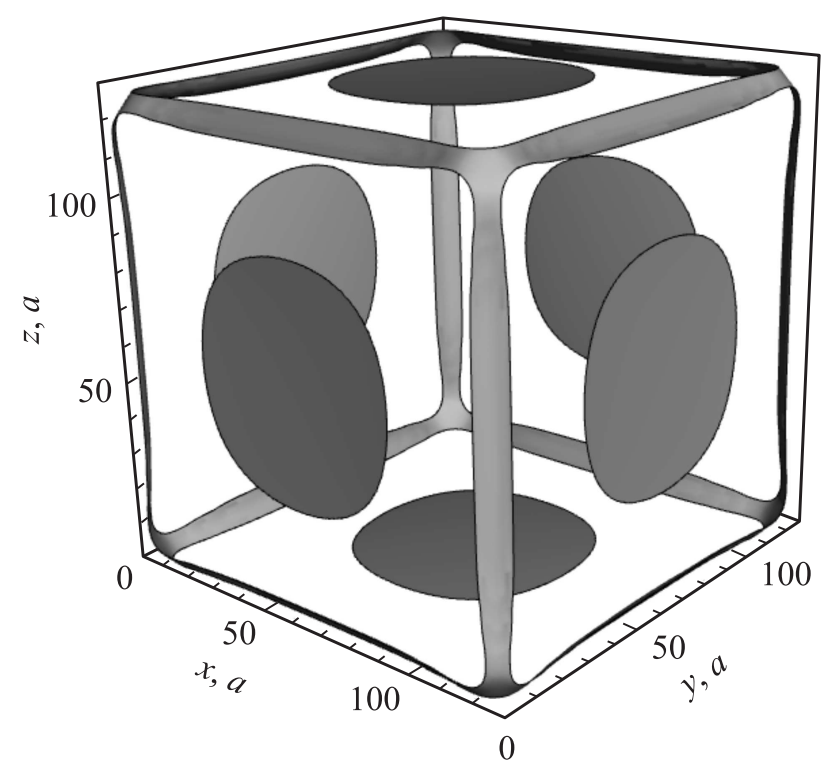

Рис. 3. Поверхности постоянной концентрации $(c=20$ at.\%) в случае обогащения зернограничной области атомами растворенного компонента в бинарном сплаве $\left(T^{*}=0.65\right.$, $c_{M}=11$ at.\%, $\left.\Delta=-0.5\right)$ в момент времени $\tau=406$. 
становится наиболее выраженным на участках, где влияние оказывают одна или несколько граней, т. е. вдоль граней (кривая 2) или ребер куба (кривая 3).

На рис. 3 приведено распределение концентрационного поля для бинарного сплава $\left(T^{*}=0.65, c_{M}=11\right.$ at.\%, $\Delta=-0.5)$, состояние которого относится к области I на рис. $1, b$ и располагается вблизи кривой бинодали $l$ (рис. $1, b)$. В этом случае происходит обогащение зернограничной области атомами растворенного компонента. Как следует из рис. 3, область повышенной концентрации, главным образом, прилегает к ребрам и вершинам куба, что соответствует случаю обогащения зернограничной области атомами сорта $B$. По мере заполнения данных областей атомами растворенного вещества происходит его сегрегация и на других участках (для рассматриваемой модели - на гранях куба). Таким образом, как обогащение примесями области, прилегающей к границе зерен, так и ее обеднение должны иметь наибольшее значение вблизи границ, имеющих наибольшую кривизну.

Наличие режимов обогащения и обеднения зернограничной области в целом согласуется с результатами работы [14], где процесс перераспределения примеси вблизи границ зерен рассматривался на основе уравнения диффузии.

6.2. 3 ернограничная преципитация. На pис. 4 приведены результаты расчета эволюции концентрационного поля для бинарного сплава, соответствующего области II на рис. $1, b\left(T^{*}=0.65, c_{M}=11\right.$ at.\%, $\Delta=-0.2)$. Данное состояние находится ниже критического значения $T_{C G B}^{*}=1+\Delta$ и для него за счет возникновения потока атомов растворенного компонента из объема зерна на его границу возникают условия для формирования второй фазы. В данном случае формируются

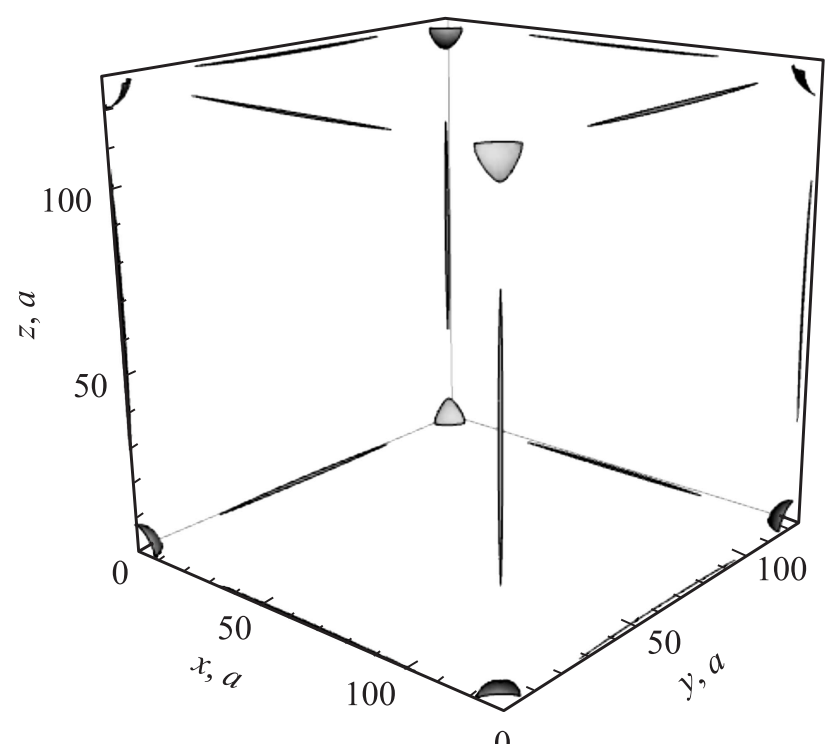

0

Pис. 4. Поверхности постоянной концентрации $(c=20 \mathrm{at} \%)$ в случае образования массивного выделения второй фазы вблизи наиболее эффективных стоков в бинарном сплаве $\left(T^{*}=0.65\right.$, $c_{M}=11$ at.\%, $\left.\Delta=-0.2\right)$ в момент времени $\tau=406$.
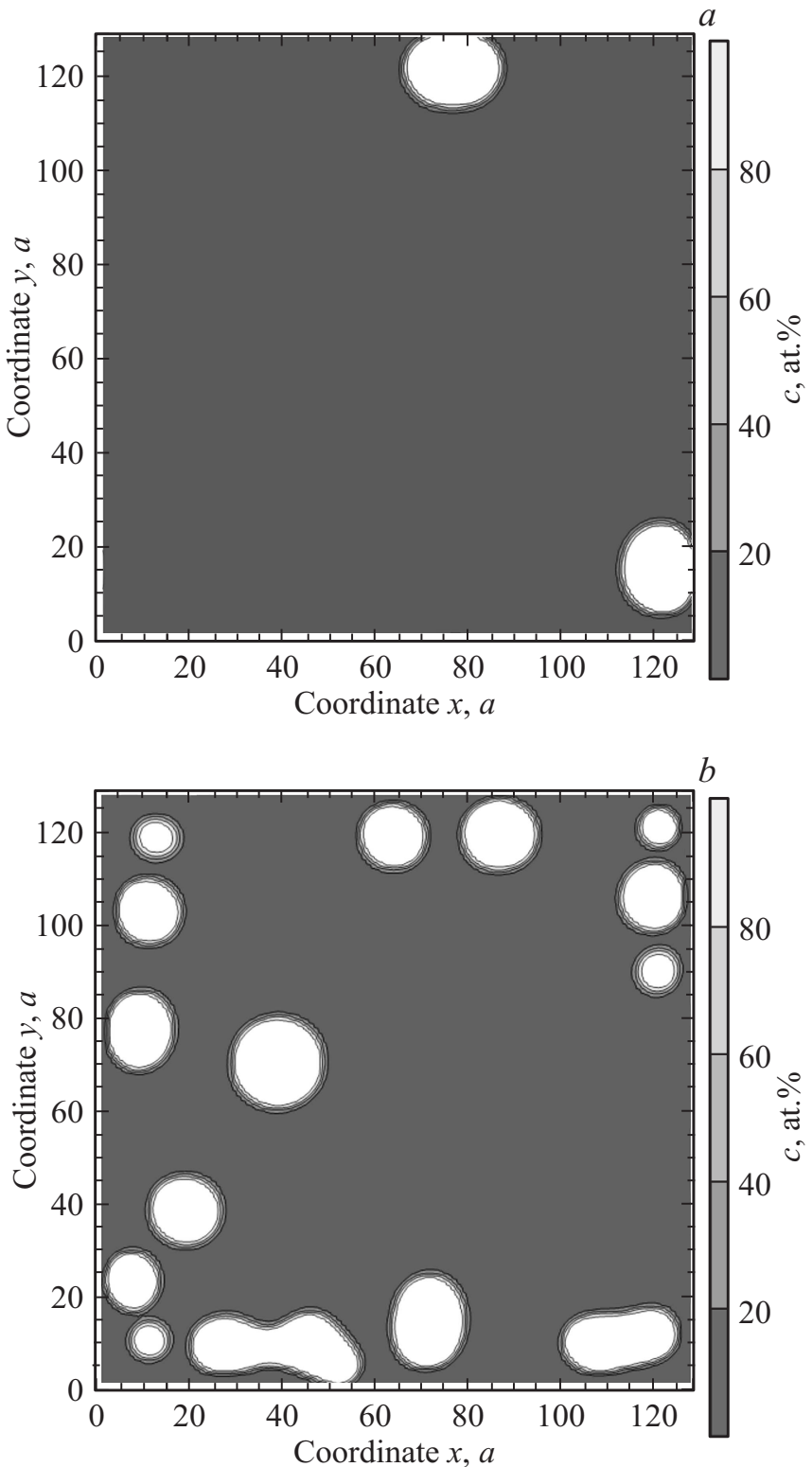

Рис. 5. Распределения концентрационного поля в случае преимущественной преципитации вблизи границ зерен для бинарного сплава $\left(c_{M}=17\right.$ at.\%, $\left.T^{*}=0.73, \Delta=0.5\right)$ в момент времени $\tau=406$. Распределение фаз приведено в для плоскостей $z=40$ (рис. $1, a)$ и $z=120$ (рис. 1.b).

достаточно массивные выделения второй фазы, которые образуются в области с наименьшим значением параметра взаимодействия $\Omega$, т.е. у вершин куба, которые представляют собой наиболее эффективные стоки для атомов сорта $B$. При этом также наблюдается некоторое обогащение атомами данного компонента области вблизи ребер куба.

Для положительных значений $\Delta$ в областях II и III на рис. 1, $a$ становится возможным образование выделений второй фазы в зернограничной области. Если рассматриваемые состояния оказываются достаточно близкими к линии бинодали 1 , то формирование выделений второй 
фазы в объеме зерна не наблюдается. При этом имеет место преимущественное образование выделений вблизи границ зерен. В области II концентрация образующихся выделений оказывается невелика. Существенное изменение происходит при приближении к линии спинодали 4 или при переходе к области III на рис. 1,a. Количество образующихся в этом случае выделений второй фазы становится заметно больше, чем в области II, что связано с переходом к безактивационному (спинодальному) механизму зарождения второй фазы. На рис. 5, $a, b$ приведены результаты расчета распределения фаз для случая $\Delta=0.5, T^{*}=0.73, c_{M}=17$ at.\%. Как следует из рисунка, выделения второй фазы образуются вблизи всей зернограничной области, т.е. как вблизи ребер и вершин куба (рис. 5, $a, b$ ), так и вблизи граней (рис. $5, b)$. Выделения второй фазы в объеме зерна для рассматриваемых параметров моделирования полностью отсутствовали (см. рис. 5, a). Данный тип распределения выделений второй фазы заметно отличается от рассмотренного выше случая отрицательных значений $\Delta$, для которого выделения формируются непосредственно на границе раздела (см. рис. 4) вблизи наиболее эффективных стоков для атомов сорта $B$.

Таким образом, несмотря на отток атомов растворенного компонента от зернограниченой области при $\Delta>0$, происходит преимущественная преципитация вблизи границ зерен, что может быть пояснено следующим образом. „Вытесненные“ при формировании обедненной области атомы сорта $B$ приводят к локальному повышению концентрации вблизи границ зерен, что снижает работу образования зародыша и заметно облегчает формирование выделений второй фазы в данной области. Наличие области, обогащенной атомами $B$ при $\Delta>0$, может быть также обнаружено на рис. 2, где все приведенные концентрационные профили имеют максимумы на расстоянии $\sim 8-10 a$ от границ зерен.

6.3. Смешанные режимы распределения компонентов сплава. Из рассматриваемой модели следует также существование различных смешанных режимов, когда вклады объемной части зерна и зернограничной области сопоставимы между собой. Проявление смешанных режимов распределения компонентов раствора наблюдается, если рассматриваемые состояния приближаются к линиям спинодали, соответствующим объему зерна (кривые 3 на рис. 1, $a$ и $b$ ). Проведем анализ некоторых из них.

Рассмотрим эволюцию фрагмента сплава, исходное состояние которого относится к области I на рис. $1, b$ $\left(T^{*}=0.65, c_{M}=15\right.$ at.\%, $\left.\Delta=-0.5\right)$. В данном случае реализуется смешанный режим, сочетающий преципитацию растворенного компонента в объеме зерна и зернограничную сегрегацию. На рис. 6 отображены результаты моделирования распределения состава бинарного сплава с приведенными параметрами. Как следует из рис. 6, в объеме зерна наблюдается формирование выделений второй фазы, которые имеют сферическую форму. Приток атомов сорта $B$ в зернограничную область обеспечивает заметное повышение концентрации

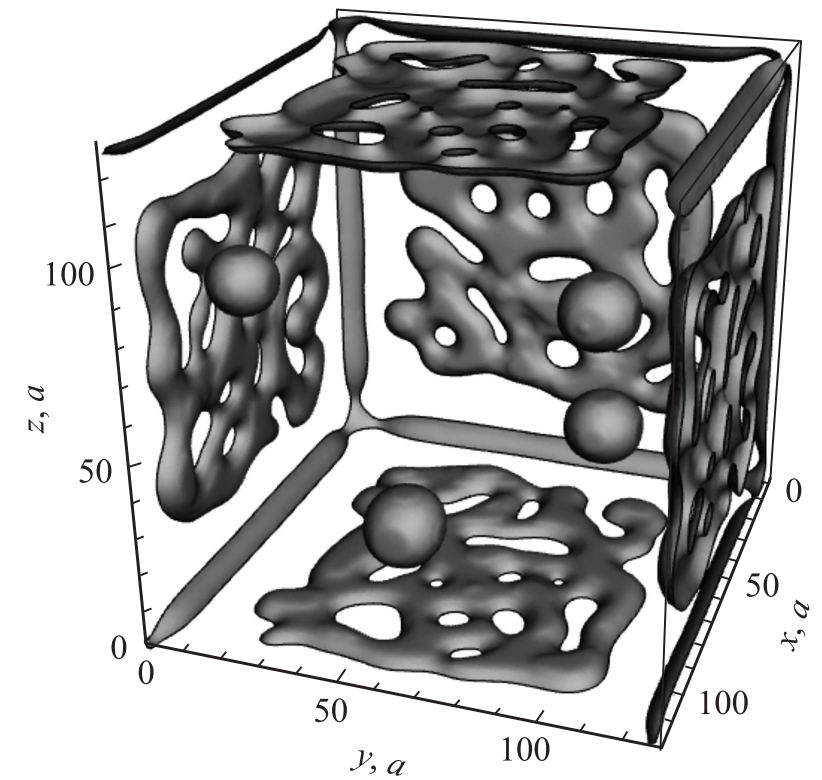

Рис. 6. Распределение фаз в бинарном сплаве при наличии смешанного механизма распада на границе и в объеме зерна $\left(c_{M}=15\right.$ at.\%, $\left.T^{*}=0.65, \Delta=-0.5\right)$ в момент времени $\tau=406$. Распределение фаз представлено поверхностями постоянной концентрации $c=50$ at.\%.

вблизи стоков (ребер и вершин куба), а также формирование перколяционной структуры, обычно свойственной спинодальному распаду. При этом образовавшиеся выделения на гранях куба имеют плоскую геометрию, их толщина примерно соответствует параметру $\delta \sim 4 a$ (см. рис. 6).

Необходимо отметить существенное отличие в формировании выделений второй фазы на границах зерен от случая однородного сплава. Как следует из рис. $1, b$, рассматриваемый набор параметров соответствует температуре $T^{*}>T_{C G B}^{*}$, т.е. выделения второй фазы на границе должны были бы полностью отсутствовать при любых значениях состава. Данная особенность, в первую очередь, связана с зависимостью параметра взаимодействия $\Omega$ от координат. По мере приближения к границе зерна параметр $\Omega$ изменяется от $\Omega_{0}$ до $\Omega_{G B}$, что соответствует постепенному изменению критической температуры от $T_{C}^{*}=1$ до $T_{C G B}^{*}=1+\Delta$, которое достигается в вершинах куба. При этом имеющиеся промежуточные значения могут предполагать существование выделений второй фазы (например, на гранях куба). Таким образом, в рассмотренном случае (рис. 6) приток вещества и наличие зависимости параметров взаимодействия от координат привели к локальному преодолению границы метастабильности и к смене механизма формирования второй фазы на границе зерна.

Еще один случай смешанного режима распределения компонентов сплава может наблюдаться для положительного значения $\Delta>0$. По мере приближения к линии спинодали 3 (рис. 1,a) характер распада несколько изменяется. Концентрации выделений второй фазы, образующихся вблизи границ и в объеме зерна, становятся 

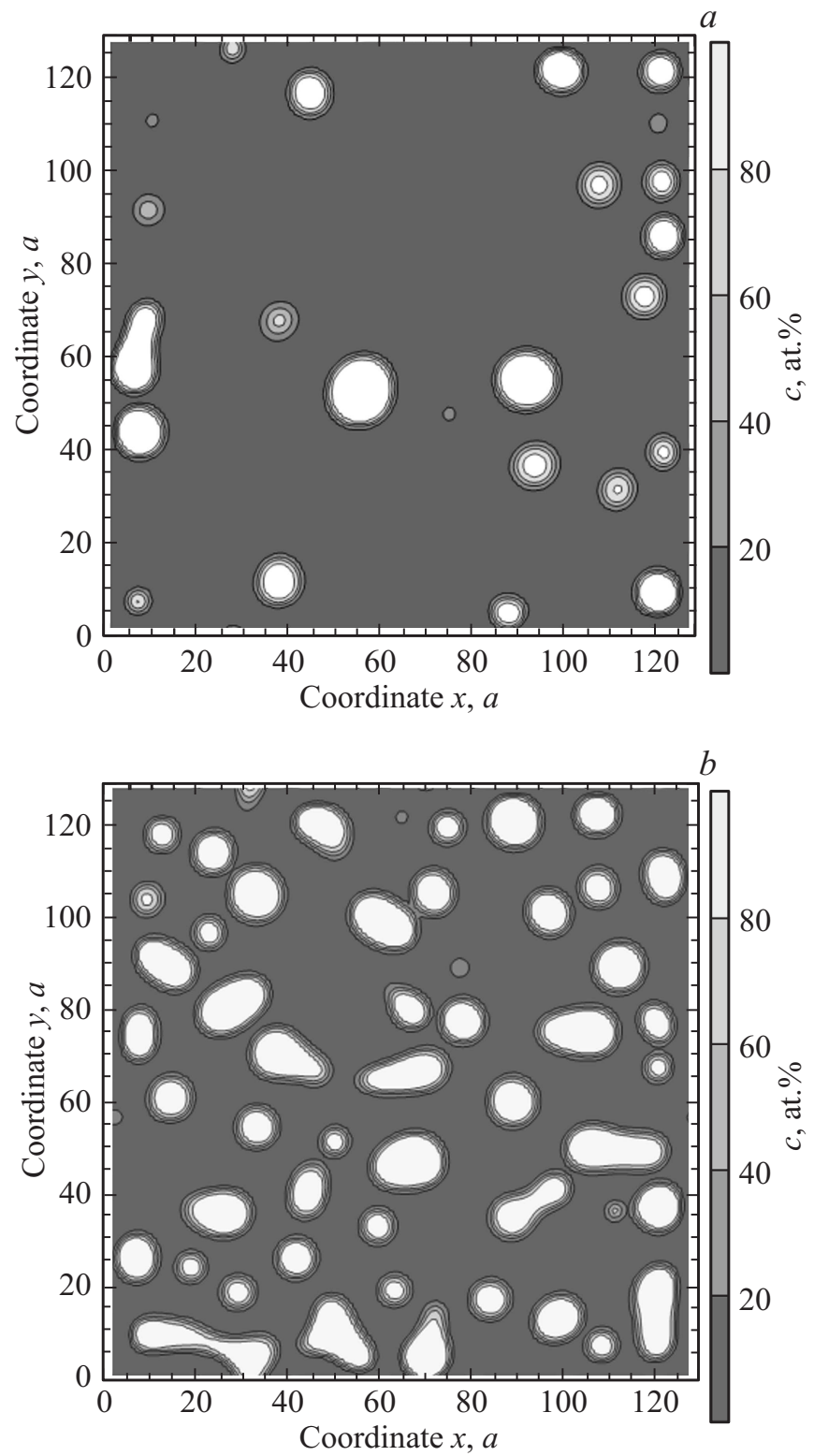

Рис. 7. Распределение концентрационного поля в случае конкурентной преципитации на границе и в объеме зерна в бинарном сплаве $\left(c_{M}=20\right.$ at. $\left.\%, T^{*}=0.73, \Delta=0.5\right)$ в момент времени $\tau=406$. Часть $a$ отражает распределение концентрационного поля в объеме зерна (в плоскости $z=64)$, часть $b-$ вблизи границы зерна (в плоскости $z=124)$.

сопоставимыми. Система переходит в режим конкурентной преципитации, при которой формирование выделений второй фазы вблизи границы зерна преобладает. На рис. 7 приведены результаты расчета распределения фаз в случае конкурентной преципитации в объеме и на границе зерна для случая, соответствующего области III на рис. $1, a\left(\Delta=0.5, T^{*}=0.73, c_{M}=20\right.$ at.\%). Так как $\Delta>0$, то вблизи границы зерна формируется обедненная область, рядом с которой образуются выделения второй фазы (аналогично рис. 5). Как следует из результатов моделирования, наиболее быстро обра- зуются выделения второй фазы вблизи границ зерен, затем с заметным отставанием формируются выделения второй фазы в объеме зерна. Концентрация выделений второй фазы в объеме зерна (см. рис. 7,a) оказывается в несколько раз меньше, чем вблизи границы (см. рис. 7,b). Различие параметров взаимодействия может привести и к различию механизмов роста, например, в один и тот же момент времени в объеме зерна может наблюдаться диффузионный рост, а на границе зерна коагуляция и коалесценция.

Режим конкурентной преципитации также может наблюдаться и для случая $\Delta<0$ вблизи спинодали 3 в области III на рис. 1, $b$. Для данной области фазовой диаграммы ситуация оказывается противоположной случаю, рассмотренному на рис. 7. Первыми образуются выделения второй фазы в объеме зерна, затем за счет притока атомов сорта $B$ из объема зерна постепенно происходит образование выделений второй фазы и в зернограничной области. Концентрация выделений в объеме зерна оказывается выше, чем на границе. При этом концентрация растворенного компонента в объеме зерна оказывается выше по сравнению с выделениями в зернограничной области, что согласуется с фазовой диаграммой (рис. 1, b).

6.4. Некоторые особенности протекания спинодального распада вблизи границ зерен. Процесс спинодального распада в бинарных сплавах наблюдается в области нестабильных состояний, которые ограничены на фазовой диаграмме линиями спинодали (рис. 1, $a, b$ ). Рассмотрим некоторые случаи протекания спинодального распада в бинарных сплавах с учетом влияния границ зерен на основе разработанного выше подхода.

На рис. 8 приведены результаты моделирования спинодального распада в бинарном сплаве для одного из состояний, соответствующего области $\mathrm{V}$ на рис. $1, a\left(c_{M}=50\right.$ at.\%, $\left.T^{*}=0.8, \Delta=0.5\right)$. Как следует из рис. $8, a$ и $b$, в рассматриваемом случае формирование второй фазы происходит в первую очередь на границе раздела, где достаточно быстро формируется отчетливая перколяционная структура, свойственная спинодальному распаду. Через некоторое время формируется сходная перколяционная структура и в объеме зерна (рис. 8,c и $d$ ). Различие между распределением фаз на границе зерна и в его объеме постепенно нивелируется. Единственное существенное отличие состоит в том, что на границе зерна состав фазы оказывается несколько выше, чем в объеме, что согласуется с различием кривых фазового равновесия 1 и 2 на рис. $1, a$.

В области IV на рис. $1, a(\Delta>0)$ фазовый переход на границе и в объеме зерна не наблюдался. Отсутствие фазового перехода в объеме зерна связано с превышением температурой критического значения $\left(T^{*}>1\right)$. При этом отток растворенного компонента из зернограничной области в матрицу (аналогично рис. 2) не может привести к формированию выделений второй фазы. Таким образом, для данной области фазовой диаграммы 
$a$
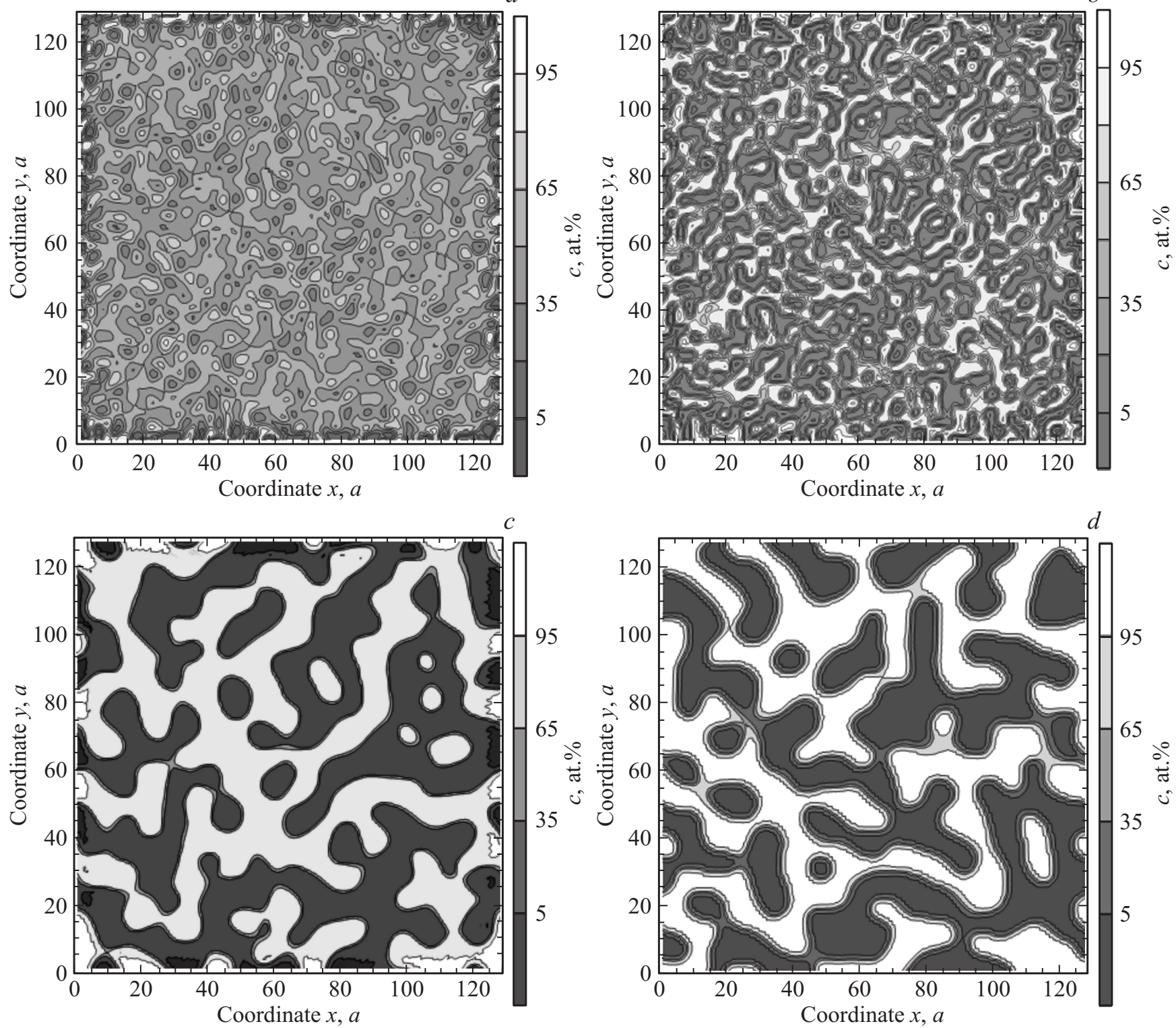

Рис. 8. Распределение концентрационного поля в случае спинодального распада в бинарном сплаве $\left(T=0.65 T_{c}, c_{M}=50\right.$ at. $\%$, $\Delta=0.5$ ) для моментов времени $\tau=5$ ( $a$ и $b)$ и $\tau=378$ ( $c$ и $d$ ). Части $a$ и $c$ соответствуют объему зерна $(z=64)$, части $b$ и $d$ соответствуют границе зерна $(z=128)$.

наблюдается только некоторое обеднение зернограничной области по атомам растворенного компонента.

В области IV на рис. $1, b(\Delta<0)$ основным механизмом формирования второй фазы является спинодальный распад, который в первую очередь протекает в объеме зерна. При этом образование второй фазы в зернограничной области не наблюдалось ввиду достаточно быстрого формирования фаз в объеме зерна и связанного с этим оттока растворенного компонента в матрицу. Вместе с тем на поздних стадиях распада происходит некоторое перераспределение компонентов сплава и на границе зерна, что связано с ростом перколяционной структуры и ее постепенным выходом в зернограничную область.
В области $\mathrm{V}$ на рис. $1, b(\Delta<0)$ происходит спинодальный распад как на границе зерна, так и в его объеме. При этом наиболее быстро происходит образование второй фазы в объеме зерна, а затем с некоторой задержкой происходит расслоение и в зернограничной области. Составы фаз на границе зерна и в его объеме отличаются друг от друга в соответствии с фазовой диаграммой рис. $1, b$ (аналогично рис. $8, c$ и $d$ ).

\section{7. Заключение}

В настоящей работе построена феноменологическая модель влияния границ зерен на распределение компонентов твердого раствора. Модель опирается на пред- 
положение о различии параметров взаимодействия в объеме зерна и вблизи его границы. Данный подход не охватывает всего многообразия процессов на границах зерен, но вместе с тем позволяет проанализировать различные особенности таких процессов как зернограничная сегрегация, преципитация и др.

Установленные особенности влияния границ зерен на распределение компонентов твердого раствора были выявлены на основе разработанной модели с использованием метода функционала плотности свободной энергии (уравнения Кана-Хилларда) с учетом зависимости параметров взаимодействия от координат. Данный подход для моделирования формирования фаз в системах с переменными параметрами взаимодействия является достаточно общим и может быть использован для моделирования других систем, содержащих различные неоднородности, взаимодействие с которыми может быть описано функцией координат.

В случае, если параметр квазихимического взаимодействия между компонентами твердого раствора на границе зерна меньше, чем в его объеме, то наблюдается зернограничная сегрегация растворенного компонента или образование массивных выделений второй фазы непосредственно на границах зерна. Процессы зернограничной сегрегации и преципитации становятся наиболее выраженными на участках границ зерен, имеющих наибольшую кривизну.

В случае, если параметр квазихимического взаимодействия на границе зерна больше, чем в его объеме, то наблюдается обеднение зернограничной области по атомам растворенного компонента. В процессе перераспределения компонентов сплава между зернограничной областью и объемом зерна могут быть созданы условия для преимущественной преципитации вблизи границ зерен. В этом случае происходит декорирование границ зерен выделениями второй фазы. Наиболее выраженным является данный процесс также на участках, где границы зерен имеют наибольшую кривизну.

Обнаружено также наличие смешанных режимов распада сплавов, для которых механизмы формирования второй фазы в объеме и вблизи границы зерна оказываются различными. Данная особенность обнаруживается, если состояния, соответствующие границе или объему зерна, оказываются вблизи границы метастабильности. В частности, наблюдался случай преимущественной преципитации в объеме зерна, которая сопровождалась зернограничной сегрегацией. При этом в процессе зернограничной сегрегации происходит формирование перколяционной структуры, свойственной спинодальному распаду. Еще одним из вариантов распада смешанного типа является конкурентная преципитация выделений второй фазы в объеме зерна и вблизи границы.

Изменение параметров взаимодействия вблизи границы зерна может оказывать влияние и в случае, если фазовый переход происходит по механизму спинодального распада. В зависимости от различия параметров взаимодействия в объеме и на границе зерна может наблюдаться опережающее протекание расслоения либо в объеме, либо на границе зерна. Составы фаз на границе и в объеме зерна могут при этом различаться.

\section{Список литературы}

[1] L. Priester. Grain Boundaries: From Theory to Engineering. Springer, Dordrecht (2013). $441 \mathrm{p}$.

[2] В.Н. Чувильдеев. Неравновесные границы зерен в металлах. Теория и приложения. Физматлит, М. (2004). 304 с.

[3] Р.А. Андриевский, А.М. Глезер. УФН 179, 337 (2009).

[4] K. Kelton, A. Greer. Nucleation in condensed matter. Elsevier (2010). 726 p.

[5] S.E. Restrepo, S.T. Giraldo, B.J. Thijsse. Mod. Simul. Mater. Sci. Eng. 21, 055017 (2013).

[6] K.C. Alexander, C.A. Schuh. Mod. Simul. Mater. Sci. Eng. 24, 065014 (2016).

[7] S. Bhattacharyya, T. Wook, H.K. Chang, L.-Q. Chen. Mod. Simul. Mater. Sci. Eng. 19, 035002 (2011).

[8] M. Javanbakht, V.I. Levitas. Phys. Rev. B 94, 214104 (2016).

[9] Б.С. Бокштейн, В.А. Есин, А.О. Родин. ФММ 109, 344 (2010).

[10] G. Kaptay. J. Mater. Sci. 51, 1738 (2016).

[11] P. Lejcek, S. Hofmann, J. Janovec. Mater. Sci. Eng. A 462, 76 (2007).

[12] Б.В. Федосеев, Е.Н. Федосеева. Письма в ЖЭТФ 97, 473 (2013).

[13] A.D. Brailsford, H.B. Aaron. J. Appl. Phys. 40, 1702 (1969).

[14] В.В. Слезов, Л.Н. Давыдов, В.В. Рогожкин. ФТТ 37, 3565 (1995).

[15] В.В. Слезов, Л.Н. Давыдов, В.В. Рогожкин. ФТТ 40, 251 (1998).

[16] M.K. Mitra, M. Muthukumar. J. Chem. Phys. 134, 044901 (2011).

[17] T. Frolov, M. Asta, Y. Mishin. Phys. Rev. B 92, 020103(R) (2015).

[18] C. Hin, Y. Brerchet, P. Maugis, F. Soisson. Acta Mater. 56, 5653 (2008).

[19] A. Umantsev. Field theoretic method in phase transformations. Springer, N.Y. (2012). 343 p.

[20] I. Steinbach. Mod. Simul. Mater. Sci. Eng. 17, 073001 (2009).

[21] J. Cahn. Acta Metall. 9, 795 (1961).

[22] П.Е. Львов, В.В. Светухин. ФТТ 58, 1382 (2016).

[23] П.Е. Львов, В.В. Светухин, К.С. Маслов. Письма в ЖТФ 42, 56 (2016).

[24] П.Е. Львов, В.В. Светухин. ФТТ 59, 345 (2017).

[25] S. Dai, Q. Du. J. Comp. Phys. 310, 85 (2016).

[26] D. Lee, J.Y. Huh, D. Jeong, J. Shin, A. Yun, J. Kim. Comp. Mater. Sci. 81, 216 (2014).

[27] D. Scheiber, R. Pippan, P. Puschnig, L. Romaner. Mod. Simul. Mater. Sci. Eng. 24085009 (2016).

[28] Л.Д. Ландау, Е.М. Лифшиц. Статистическая физика. Наука, М. (1976). Ч. 1. 584c.

[29] П.Е. Львов, В.В. Светухин. ФТТ 57, 1192 (2015).

[30] C. Zhang, M. Enomoto. Acta Mater. 54, 4183 (2006).

[31] J.W. Cahn, J.E. Hilliard. J. Chem. Phys. 28, 258 (1958).

[32] П.Е. Львов, В.В. Светухин. ФТТ 56, 1825 (2014).

[33] L.-Q. Chen, J. Shen. Comp. Phys. Commun. 108, 147 (1998).

[34] J. Zhu, L.-Q. Chen, J. Shen, V. Takare. Phys. Rev. E 60, 3564 (1999). 